\title{
Structure of a Drosophila Sigma Class Glutathione S-transferase Reveals a Novel Active Site Topography Suited for Lipid Peroxidation Products
}

\author{
Bogos Agianian ${ }^{1 *}$, Paul A. Tucker ${ }^{2}$, Arie Schouten ${ }^{1}, K_{\text {Kevin Leonard }}^{3}$ \\ Belinda Bullard ${ }^{3}$ and Piet Gros ${ }^{1}$
}

${ }^{1}$ Department of Crystal and Structural Chemistry, Bijvoet Center for Biomolecular

Research, Utrecht University Padualaan 8, $3584 \mathrm{CH}$ Utrecht The Netherlands

${ }^{2}$ EMBL Hamburg Outstation 22603 Hamburg, Germany

${ }^{3}$ European Molecular Biology Laboratory (EMBL), 69117 Heidelberg, Germany

\begin{abstract}
Insect glutathione-S-transferases (GSTs) are grouped in three classes, I, II and recently III; class I (Delta class) enzymes together with class III members are implicated in conferring resistance to insecticides. Class II (Sigma class) GSTs, however, are poorly characterized and their exact biological function remains elusive. Drosophila glutathione $S$-transferase-2 (GST-2) (DmGSTS1-1) is a class II enzyme previously found associated specifically with the insect indirect flight muscle. It was recently shown that GST-2 exhibits considerable conjugation activity for 4-hydroxynonenal (4-HNE), a lipid peroxidation product, raising the possibility that it has a major anti-oxidant role in the flight muscle. Here, we report the crystal structure of GST-2 at $1.75 \AA$ resolution. The GST-2 dimer shows the canonical GST fold with glutathione (GSH) ordered in only one of the two binding sites. While the GSH-binding mode is similar to other GST structures, a distinct orientation of helix $\alpha 6$ creates a novel electrophilic substrate-binding site (H-site) topography, largely flat and without a prominent hydrophobic-binding pocket, which characterizes the $\mathrm{H}$-sites of other GSTs. The H-site displays directionality in the distribution of charged/polar and hydrophobic residues creating a binding surface that explains the selectivity for amphipolar peroxidation products, with the polar-binding region formed by residues Y208, Y153 and R145 and the hydrophobic-binding region by residues V57, A59, Y211 and the C-terminal V249. A structure-based model of 4-HNE binding is presented. The model suggest that residues Y208, R145 and possibly Y153 may be key residues involved in catalysis.
\end{abstract}

(C) 2003 Elsevier Science Ltd. All rights reserved

Keywords: glutathione S-transferase; Drosophila; flight muscle; lipid peroxidation; 4-hydroxynonenal

\section{Introduction}

Present address: B. Agianian, EMBL, 69117 Heidelberg, Germany.

Abbreviations used: GST, glutathione-S-transferase; GSH, glutathione; PDGS, prostaglandin D synthase; $\mathrm{PGD}_{2}$, prostaglandin $\mathrm{D}_{2} ; \mathrm{PGH}_{2}$, prostaglandin $\mathrm{H}_{2}$; IFM, indirect flight muscle; 4-HNE, 4-hydroxynonenal; G-site, glutathione-binding site; $\mathrm{H}$-site, electrophilic (cosubstrate)-binding site; $\mathrm{TnH}$, "heavy" troponin; CDNB, 1-chloro-2,4-dinitrobenzol; DCNB, 1,2-dichloro-4nitrobenzene; NBD-Cl, 7-chloro-4-nitrobenzo-2-oxa-1,3diazole; EA, ethacrynic acid; MIRAS, multiple isomorphous replacement with anomalous scattering; rmsd, root mean square deviation; FOM, figure of merit PDB, Protein Data Bank.

E-mail address of the corresponding author: agianian@embl-heidelberg.de
Glutathione-S-tranferases (GSTs) are a family of multi-functional enzymes which are mainly involved in the detoxification of harmful physiological and xenobiotic electrophilic compounds, which they conjugate with glutathione (GSH). Currently, mammalian cytosolic GSTs are divided into eight classes: Alpha, Mu, Pi, Theta, Zeta, ${ }^{1,2}$ and recently Sigma, ${ }^{3}$ Kappa $^{4}$ and Omega. ${ }^{5}$ In addition, a membrane-associated microsomal GST has been identified. ${ }^{1}$ Vertebrate Sigma GSTs are GSH-dependent prostaglandin D synthases (PGDSs) that catalyze the isomerization of prostaglandin D2 (PGD2) from PGH2. ${ }^{6,7}$ Members of the Sigma family in invertebrates include the active GSTs from the squid, ${ }^{8}$ parasitic helminths ${ }^{9}$ and 
(a)

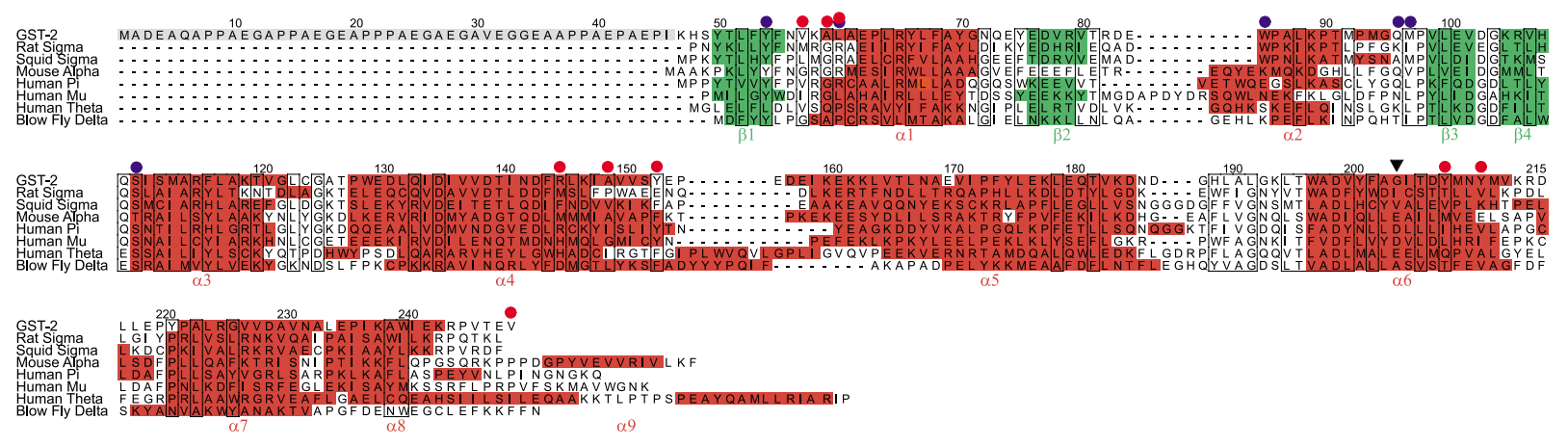

(b)

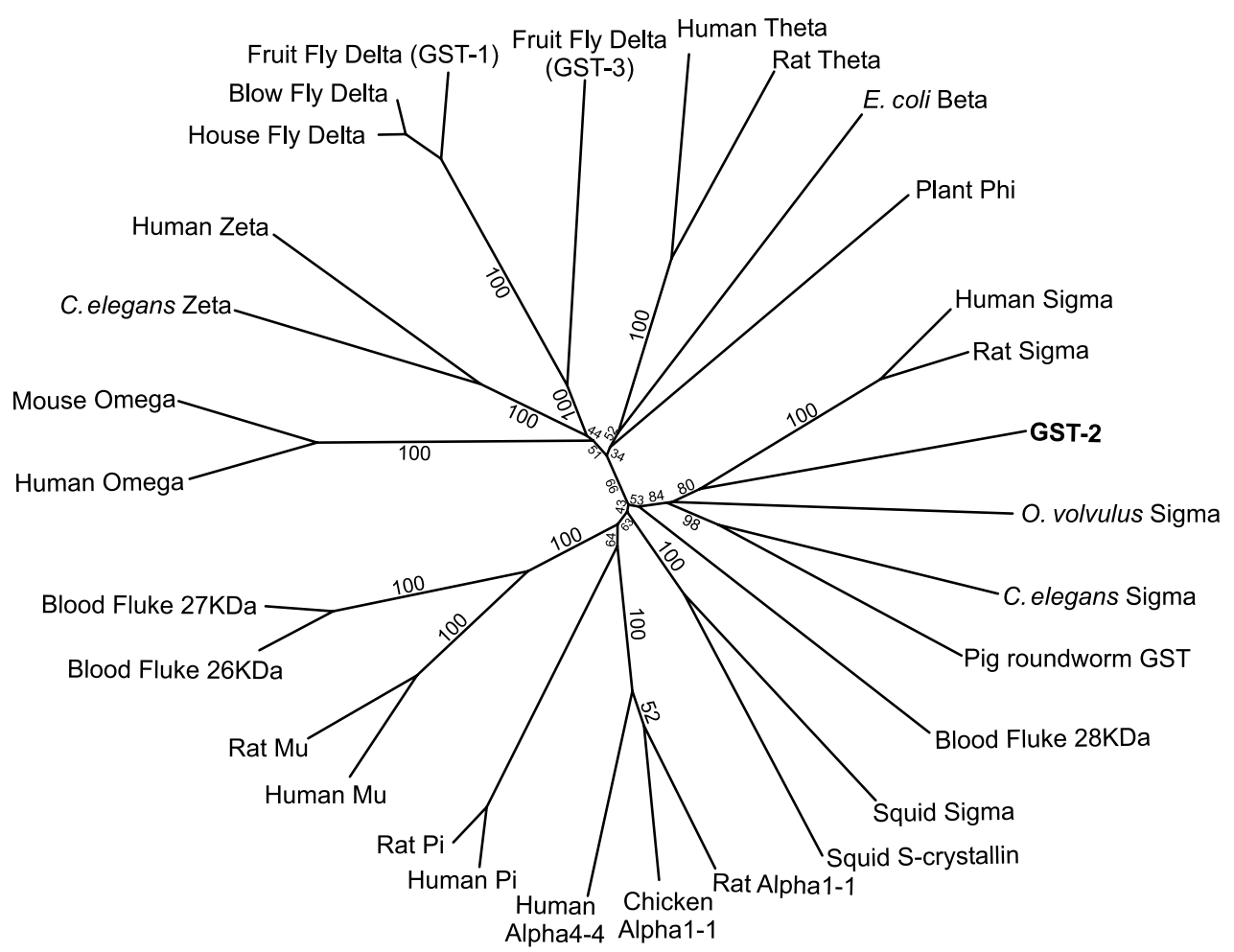

Figure 1. (a) Structure-based sequence alignment of GST-2 and GSTs of known tertiary structure. Secondary structure elements are colored red ( $\alpha$-helix) and green ( $\beta$-strand). Regions of sequence conservation according to Livingstone et $a l . .^{82}$ are boxed. The N-terminal extension of GST-2 is highlighted in gray. Residues of GST-2 forming the GSH and putative electrophilic-binding sites are marked by blue and red circles, respectively, at the top of the alignment. L60, which contacts GSH and is also a putative H-site residue, is marked with two circles. The black triangle indicates G203, the bulge-inducing residue of $\alpha 6$ helix (see the text). Amino acid sequences and secondary structure information for GSTs were obtained from the SwissProt (sw) and Protein Data Bank (PDB) as follows: rat Sigma (sw, pgd_rat; PDB, 1PD2), squid Sigma (sw, gts_ommsl; PDB, 1GSQ), mouse Alpha4-4 (sw, gta4_mouse; PDB, 1B48), human Pi (sw, gtp_human; PDB, 1PGT), human Mu (sw, gtm1_human; PDB, 1GTU), human Theta (sw, gtt2_human; PDB, 1LJR), blow fly Delta (sw, gtt1_luccu). ${ }^{32}$ (b) Unrooted phylogenetic tree showing the most likely relationship between GST-2 and representative members of other GST classes, including squid S-crystallin and GSTs from flukes and nematodes. Redundant, highly similar and partial sequences were omitted. Local bootstrap probabilities, normalized to 100, as an indicator of confidence are shown on the branches. The sequences are from the SwissProt (sw) or GenBank (gb): house fly Delta (sw, gtt1_musdo), blow fly Delta (sw, gtt1_luccu), fruit fly Delta (GST-1: sw, gtt1_drome; GST-3: gb, AF179869), human Theta (sw, gtt2_human), rat Theta (sw, gtt1_rat), E. coli Beta (gb, D38497), A. thaliana Phi (gb, D17672), human Sigma (sw, pgd_human), rat Sigma (sw, pgd_rat), O. volvulus Sigma (gb, S40165), Caenorhabditis elegans Sigma ( $\mathrm{gb}, \mathrm{Z50859),} \mathrm{pig} \mathrm{roundworm} \mathrm{(} \mathrm{gb}, \mathrm{S38626)}$ ), blood fluke-28 (sw, gt28_schma), squid Sigma (sw, gts_ommsl), squid S-crystallin (sw, sc20_ommsl), rat Alpha1-1 (sw, gta1_rat), chicken Alpha1-1 (gb, L15386), human Alpha4-4 (sw, gta4_human), human Pi (sw, gtp_human), rat Pi (sw, gtp_rat), human Mu (sw, gtm2_human), rat Mu (sw, gtm1_rat), blod fluke-26 (sw, gt26_schma), blood fluke-27 (sw, gt27_schma), human Omega (gb, AF212303), mouse Omega (gb, AB008807), C. elegans Zeta (gb, Z66560), human Zeta (gb, U86529). 
Table 1. Activity of GST-2

\begin{tabular}{|c|c|}
\hline Substrate & Specific activity ( $\mu \mathrm{mol} / \mathrm{minute}$ per $\mathrm{mg}$ ) \\
\hline 1-Chloro-2,4-dinitrobenzol (CDNB) & $0.49 \pm 0.02$ \\
\hline 1,2-Dichloro-4-nitrobenzene (DCNB) & $0.44 \pm 0.01$ \\
\hline 7-Chloro-4-nitrobenzo-2-oxa-1,3-diazole (NBD-Cl) & $2.02 \pm 0.2$ \\
\hline $\mathrm{NBD}-\mathrm{Cl}+\mathrm{TnH}(1: 2$ molar $)$ & $2.1 \pm 0.3$ \\
\hline Ethacrynic acid (EA) & $0.24 \pm 0.02$ \\
\hline Trans-2-nonenal & $1.4 \pm 0.1$ \\
\hline 4-Hydroxynonenal ${ }^{\mathrm{a}}$ & $8.4 \pm 0.4$ \\
\hline
\end{tabular}

nematodes, ${ }^{10}$ and the enzymatically inactive $S$-crystallins from the eye lens of cephalopods. ${ }^{11}$

Insect GSTs fall into three distinct classes, I, II ${ }^{12}$ and III. ${ }^{13}$ Class I enzymes have been classified in an insect-specific Delta class. ${ }^{2}$ Class II GSTs have been identified in dipteran insects including Drosophila melanogaster, ${ }^{14}$ Musca domestica ${ }^{15}$ and Anopheles gambiae ${ }^{16}$ and are mainly members of the Sigma class. Sequence analysis of the D. melanogaster genome identified no Alpha, Mu or Pi classes. ${ }^{17}$ According to a commonly used nomenclature, to which we adhere here for consistency, the insect Delta and Sigma GSTs are termed GST-1 and GST-2, respectively. The Delta class enzymes (as well as class III enzymes) have been implicated in detoxification, in particular in conferring resistance towards various insecticides ${ }^{18,19}$ and use a serine as the catalytic residue. Insect Sigma class GSTs are poorly characterized and have a catalytic tyrosine (this work).

In D. melanogaster, GST-2 (proposed new name: DmGSTS1-1 ${ }^{17}$ ) has marginal sequence homology to the GST-1 isoenzyme, suggesting that the two classes have discrete biological functions. This is supported by the fact that GST-2 carries a peculiar proline/alanine rich $\mathrm{N}$-terminal extension and that it is almost exclusively localized on the thin filaments of the indirect flight muscles (IFMs) ${ }^{20}$ in interaction with troponin- $\mathrm{H}(\mathrm{TnH}) .{ }^{21}$ Based on its close association with the troponin complex it was hypothesized that Drosophila GST-2 may play a structural role in the IFMs. ${ }^{20}$ Until recently the catalytic properties of GST-2 were not documented; a recent study, however, demonstrated that the enzyme has very low activity for typical GST substrates, but is significantly active in catalyzing the conjugation of GSH with the lipid peroxidation product 4-hydroxynonenal (4-HNE). ${ }^{22}$ 4-HNE is produced as a by-product of lipid peroxidation under conditions of oxidative stress and has physiological effects in signaling, cell proliferation, differentiation $^{23}$ and apoptosis, ${ }^{24}$ as well as being cytotoxic. ${ }^{25}$ Thus, the newly discovered substrate specificity of GST-2 raises the possibility that this enzyme may contribute significantly in the protection of the muscle against harmful effects of 4 -HNE. Such a function would be analogous to the one performed by the Alpha4-4 GSTs in mammals, a specialized Alpha subclass with exceptionally high activity towards $4-\mathrm{HNE} .^{26,27}$
Structures of GSTs from mammalian classes have been described (see references in Sheehan et al. ${ }^{28}$ ). Non-mammalian GST structures include ones from the bacterial Beta class ${ }^{29}$ and the plant Phi and Zeta classes. ${ }^{30,31}$ Currently, only three structures of insect GSTs are known, which all belong to the Delta class: one from the Australian sheep blowfly Lucilia cuprina ${ }^{32}$ and two from two closely related isoforms from the mosquito Anopheles dirus. $^{33}$

\section{Results}

\section{Homology with other GST family members}

A sequence alignment between GST-2 and representative members of GST classes was used to create an unrooted phylogenetic tree (Figure 1(b)). The tree clearly shows that GST-2 is distinct from GSTs of the insect Delta class and confirms its grouping within the Sigma class. Interestingly, GST-2 is most identical with the vertebrate PGDSs $^{7}$ and moderately similar to GSTs from nematodes and the helminth Schistomoma mansoni (blood fluke). Sequence identities between GST-2 and PGDSs range between 40 and 50\% whereas the figure drops to $35-45 \%$ for the closest invertebrate homologs. GST-2 shows weak similarity to the $S$-crystallins from cephalopods and the GSTs from the mammalian classes $(<30 \%)$ and very low identity to the insect Delta class members $(<20 \%)$, including its homologs GST-1 and GST-3 from D. melanogaster. GST-2 (together with its Musca ortholog) features a proline/alanine-rich N-terminal extension (45-residues); such N-terminal extensions, albeit shorter, are observed only in Omega class GSTs and in a Sigma GST from Onchocerca volvulus.

\section{Catalytic activity}

Recently, Singh et al. ${ }^{22}$ investigated aspects of the catalytic activity of GST-2; they found that recombinant or native GST-2 had significant glutathione-conjugating activity towards 4-HNE, whereas the activity was low for typical GST substrates such as aryl-alkyl halides, epoxides and nitro-aromatic compounds. We measured the catalytic activity of the recombinant GST-2 used in 
(a)

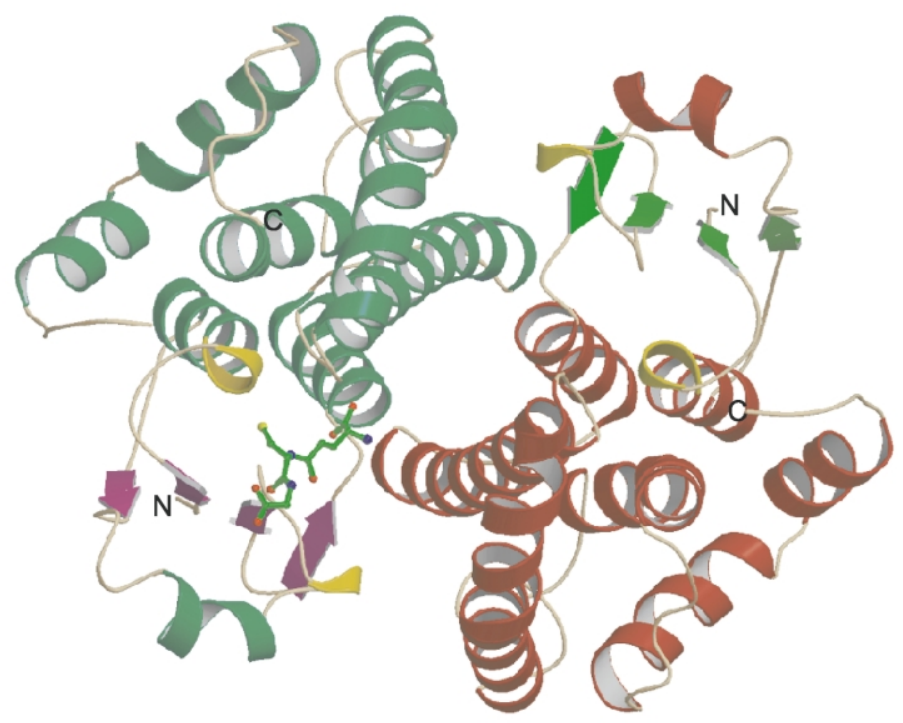

(b)

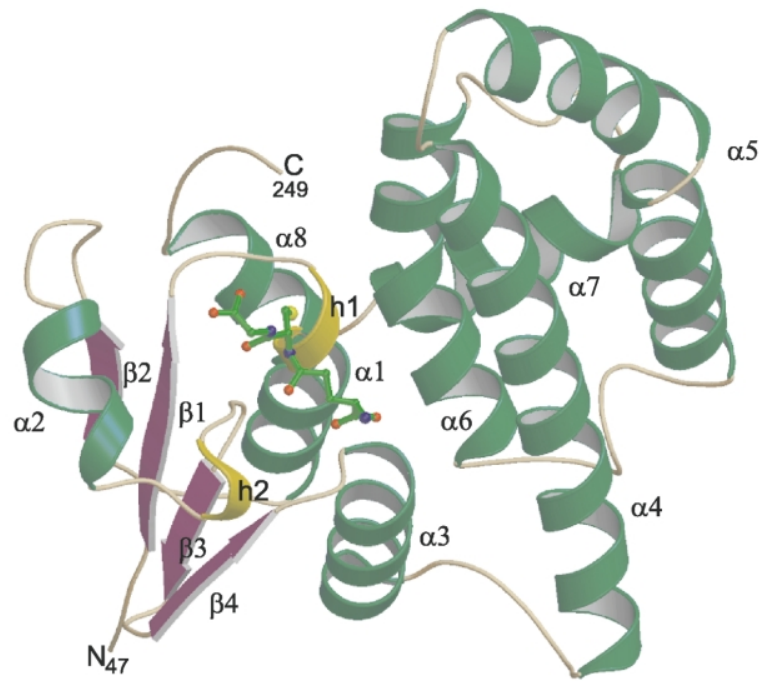

Figure 2. Structure of GST-2. (a) The GST-2 dimer viewed down the 2-fold axis. The $\mathrm{N}$ and $\mathrm{C}$ termini are indicated. The GSH molecule bound to monomer $\mathrm{A}$ is shown in ball and stick representation. (b) Monomer A in a ribbon diagram illustrating the secondary structure elements and the bound GSH molecule. Helices are colored green, $\beta$-strands magenta and $3_{10}$ helices gold. Naming follows standard nomenclature from other GST structures.

our structure determination (Table 1). Our results, which confirm the observations by Singh et al.,2 show that GST-2 has low but detectable activities for CDNB, DCNB and EA, which are usually good substrates for members of the $\mathrm{Pi}, \mathrm{Mu}$ and Alpha classes. In contrast, GST-2 displays considerable activities towards trans-2-nonenal, an $\alpha, \beta$-unsaturated carbonyl compound derived from secondary lipid-peroxidation, and, surprisingly, the aryl halide NBD-Cl a preferential substrate for the Alpha and microsomal isozymes. ${ }^{34}$ GST-2 localizes to the IFMs via interactions with the hydrophobic C-terminal tail of $\mathrm{TnH} .^{20}$ To assess the potential influence of $\mathrm{TnH}$ on the enzymatic activity of GST-2, assays using NBD-Cl were performed in the presence of excess TnH. As shown in Table 1, $\mathrm{TnH}$ had no significant effect on the specific activity. This complies with the observation that truncation of the N-terminal extension of GST-2, which is homologous to the tail of $\mathrm{TnH}$ and could potentially act similarly, does not notably influence the kinetic parameters of the enzyme. ${ }^{22}$

\section{Overal structure of GST-2}

Despite the low sequence identity with GSTs of known structure, GST-2 adopts the canonical GST fold (Figure 2). The secondary structure elements compared to other GSTs are conserved in a structure-based alignment (Figure 1(a)) with the exception of helix $\alpha 9$, which is characteristic of the Alpha and Theta classes. Two subunits related by a non-crystallographic 2-fold axis form a dimer with dimensions of about $60 \AA \times 50 \AA \times 50 \AA$. Each monomer is formed by two distinct domains; a small thioredoxin-like $\mathrm{N}$-terminal domain (residues 47-120) and a larger helical C-terminal domain (residues 121-249), with a wide interdomain cleft where the catalytic site resides. The $\mathrm{N}$-terminal domain is formed by a four-stranded $\beta$-sheet flanked on one side by helices $h 1 / \alpha 1$ (residues 59-71) and $\alpha 3$ (residues 110-120) and on the other by helix $\alpha 2$ (85-95) together with the short $3_{10}$ helix h2 (93-95). These structural elements are arranged in a $\beta \alpha \beta \alpha \beta \beta \alpha$ motif in 
which $\beta 3$ is antiparallel with respect to the other $\beta$-strands. P98, at the end of the loop connecting helix h2 and strand $\beta 3$, is in cis conformation. Equivalent cis-residues have been found in all GST structures known. ${ }^{35}$ The five helices of the C-terminal domain make an $\alpha$-helical bundle. A 3D-superposition of the $C^{\alpha}$ trace of GST-2 with representative structures from other GST classes was performed (not shown). As expected, the highest degree of structural homology was achieved with the vertebrate and squid Sigma structures (rmsd: $1.41 \AA / 171$ residues and $1.69 \AA$ / 188 residues, respectively), followed by the human Alpha4-4 (rmsd: $1.64 \AA / 119$ residues) and $\mathrm{Mu}$ (1.69 A/161 residues) members. As observed before in GST-structure comparisons, superposition of the $\mathrm{N}$-terminal domains revealed big differences in the orientation of the C-terminal domains. The most dramatic shift is observed with the C-terminal domain of the mammalian Alpha4-4 enzymes (rmsd $\mathrm{C}^{\alpha}$ atoms $\sim 8 \AA$ ).

\section{Inter-domain and inter-subunit interactions}

The $\mathrm{N}$ and C-terminal domains are held together through packing of helices $\alpha 1$ and $\alpha 3$ against helices $\alpha 6$ and $\alpha 4$ and with interactions of helix $\alpha 8$ with parts of $\alpha 1, \beta 1$ and $\beta 2$. These interactions are predominantly hydrophobic in nature with a relatively small number of hydrogen bonds. The short C-terminal extension is rigid, stabilized by van der Waals contacts and main-chain hydrogenbonding. These contacts orient the $C$ terminus in such a way that it does not block the entrance to the catalytic site and ensure that residue V249 lines up in a continuous surface with residues forming the floor of the putative H-site (see Figure 4(b)).

The GST-2 dimer is stabilized mainly by interactions of helices $\alpha 3$ and $\alpha 4$ from each subunit, which form a bundle around the 2-fold axis (Figure 2 ). The majority of the contacts are water-mediated with some hydrophobic interactions, the most prominent being the stacking of W129 against F116, L105 and V102. The hydrophobic "ladder" from symmetry-related residues adjoining the monomers, typical of other GST structures (e.g. plant structures $^{36}$ ) is not observed. Only residues at the bottom one third of the long helix $\alpha 4$ are interacting at the dimer interface, resulting in the formation of an open V-shape. The shortest $C^{\alpha}-C^{\alpha}$ distance of the opening between the subunits along the helical towers is $\sim 6 \AA$. At the center of the interface, R115 of one subunit stacks with the symmetry-related arginine from the second subunit. The structure of mGSTA $4-4,{ }^{37}$ which exhibits high conjugation activity for 4-HNE, showed that similarly stacked arginine residues R69 and R15 are not symmetry-related and are signaling substrate binding across the dimer interface. Such a signaling mechanism is not suggested by the GST2 structure. GST-2 displays an inter-subunit hydrophobic "key-lock" motif whereby M94 inserts into a hydrophobic pocket created by residues T140,
F174, Y175, K178 and L179. This motif is absent in the squid Sigma enzyme, ${ }^{38}$ but it is present in the vertebrate Sigma isoenzyme, ${ }^{6}$ while it is degenerate in the insect Delta. ${ }^{32}$ The buried area at the dimer interface is $1790 \AA^{2}$. This number shows a limited inter-subunit contact most similar to the one in the Omega class, ${ }^{5}$ compared with usually 2700$3000 \mathrm{~A}^{2}$ in mammalian GSTs.

\section{The G-site}

GSH was modeled only in monomer A (see Materials and Methods) bound in an extended conformation to a groove defining the G-site. The ligand is oriented so that the $\gamma$-glutamyl moiety points towards the center of the dimer while the glycyl part is facing outwards. Almost all residues defining the G-site display excellent electron in omit maps (Figure 3(a)), while the density for GSH is weaker. Most of the GSH-protein contacts involve hydrogen-bonding of polar atoms of the ligand and only minor hydrophobic interactions (Figure 3(b)). The polar interactions, however, are not extensive with all amide nitrogen atoms and the glycyl carboxyl oxygen not involved in any hydrogen-bonding. GSH contacts with the B subunit are not observed, as is the case in other GSH-GST complex structures including the insect Delta. GSH-mediated subunit interactions are, however, found in the Sigma GSTs from the squid $^{38}$ and vertebrates, ${ }^{6}$ in which an acidic residue (D101 in the squid and D97 in the vertebrateSigma) forms a salt-bridge with the terminal amide nitrogen of GSH. The equivalent Asp residue in GST-2, D143 (Figure 1(a)), is instead hydrogen-bonded to Q109, which adopts unfavorable stereochemistry as described for the equivalent residues in most GST structures. ${ }^{39}$ The catalytic residue of GSTs is usually a tyrosine with the exception of the Beta and Omega structures where it is a cysteine and the insect Delta, plant Phi and Zeta GSTs where it is a serine. The GST-2 structure shows that the equivalent catalytic residue is Y54 (Figure 3).

\section{The H-site}

The putative H-site of GST-2 is largely formed by the top sections of helices $\alpha 4$ and $\alpha 6$, the loop connecting $\beta 1$ and $\alpha 1$ and the $C$ terminus of the protein (Figure 4(b)). A surface representation of the H-site shows a shallow largely flat surface that constitutes a novel topography (Figure 4(b)). Y153 is the only $\mathrm{H}$-site residue that shows significant variability in its position between monomer $\mathrm{A}$ and $B$, based on a superposition of the two N-terminal domains. Specifically, the phenyl ring of Y153 is displaced sidewise towards V249 in monomer B compared to monomer $\mathrm{A}\left(\mathrm{O}^{\eta}-\mathrm{O}^{\eta}\right.$ distance $\left.\sim 2 \AA\right)$, away from GSH $\left(\mathrm{O}^{n}-\mathrm{S}\right.$ distance $7.5 \AA$ and $6.9 \AA$ in monomer $\mathrm{B}$ and $\mathrm{A}$, respectively). This movement, however, which is the result of the flexibility of the loop between $\alpha 4$ and $\alpha 5$ which adopts different 
(a)
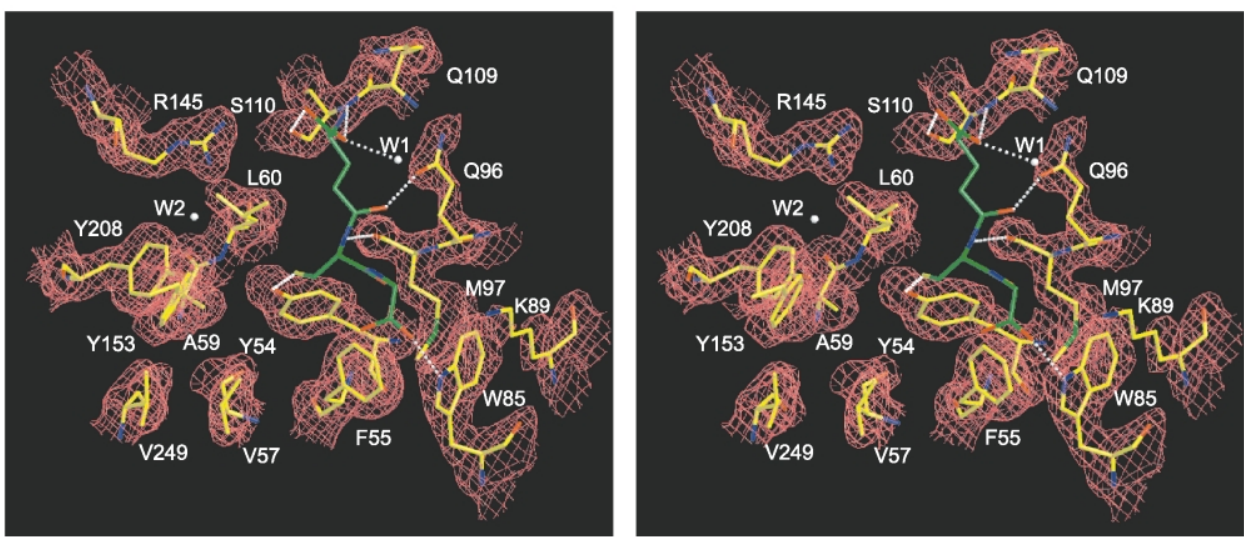

(b)

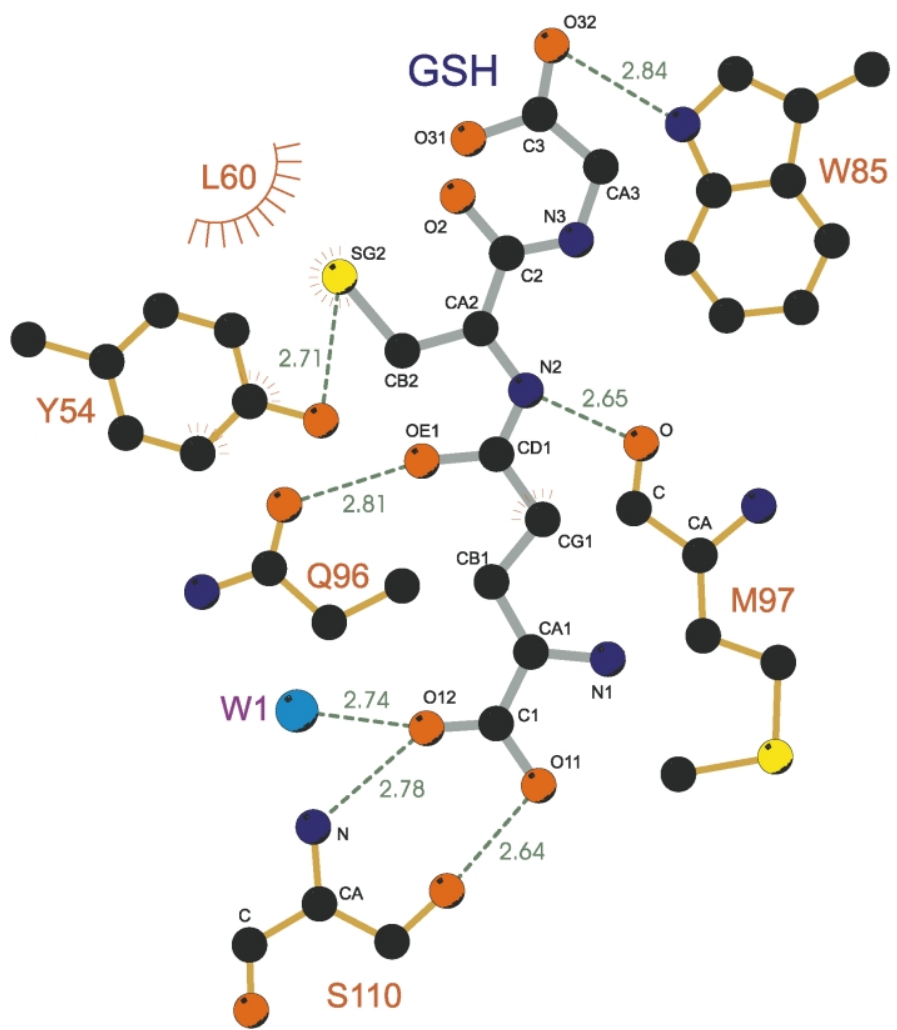

Figure 3. GSH-binding to GST-2. (a) Stereo view showing the G-site residues including H-site residues in the vicinity (see the text). The electron density corresponds to a $2 F_{\mathrm{o}}-F_{\mathrm{c}}$ omit map contoured at $1.2 \sigma$. GSH is drawn as a stick model with carbon atoms colored green, nitrogen atoms blue and oxygen atoms red. Hydrogen bonding interactions of GSH are shown by broken lines. The G and $\mathrm{H}$-site water molecules $\mathrm{W} 1$ and $\mathrm{W} 2$, respectively, are shown. (b) Schematic representation showing details of GSH contacts. The Figure was plotted using LIGPLOT. ${ }^{83}$

structures in the two subunits (rmsd $\mathrm{C}^{\alpha}$ atoms, $\sim 2.2 \AA$ ), does not essentially alter the overall shape of the H-site. The hydroxyl oxygen of Y208 is $5.3 \AA$ apart from the sulfur atom of GSH and participates in a water-mediated hydrogen bond with nitrogen $\mathrm{N}^{n}$ of R145. The water molecule involved (W2; Figure 3(a)) is found in that position in both monomers and could be implicated in the conjugation reaction mechanism as suggested for similar hydration water molecules in $\mathrm{Pi}$ class structures $^{39,40}$ as well as for the conjugation of
GSH to 4-HNE by the Alpha4-4 subclass. ${ }^{41,42}$ Similarly positioned water molecules were observed in the insect AdGST1-3 structure (but not in the Lucilia or AdGST1-4 ones) $)^{33}$ and in the murine Alpha4-4 structure. ${ }^{37}$ The guanidino group of R145 is $6.6 \AA$ away from the sulfur atom of GSH. The proximity of this arginine suggests that it might be playing a direct or indirect role in catalysis.

Of particular importance for the creation of the unique flat topography of the $\mathrm{H}$-site is residue Y208, which is "filling up" the space that is usually 
(a)

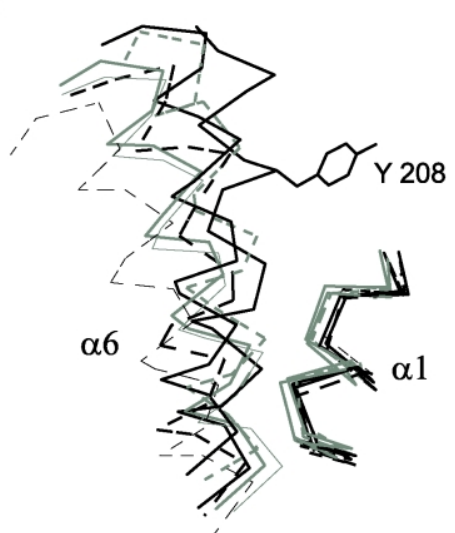

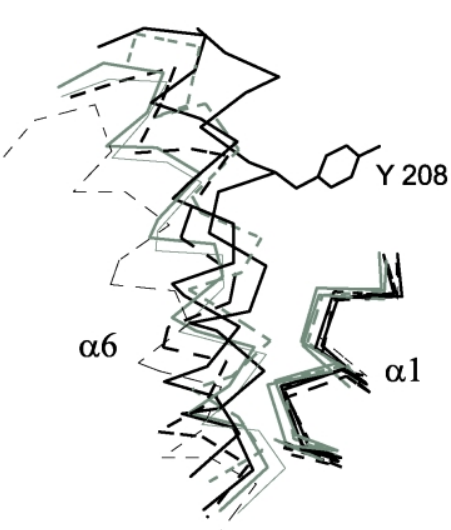

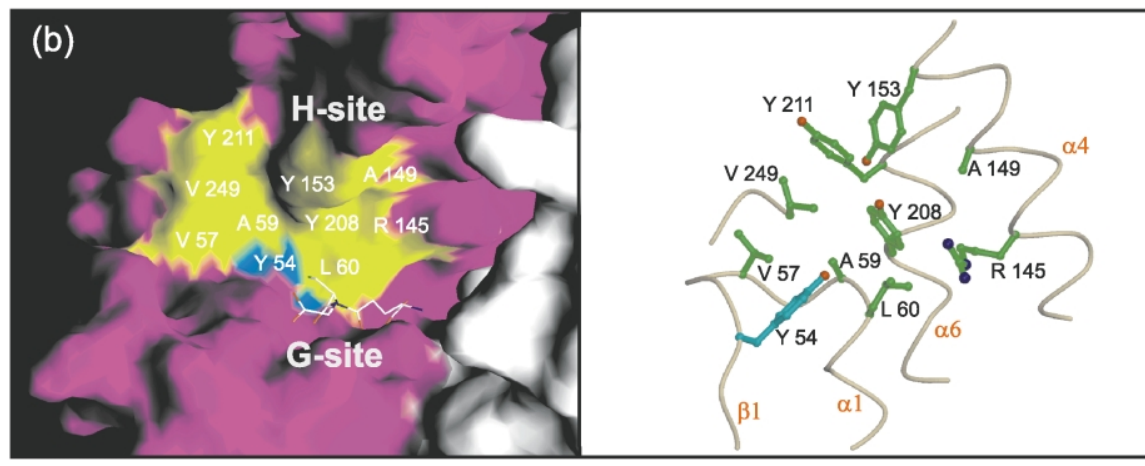

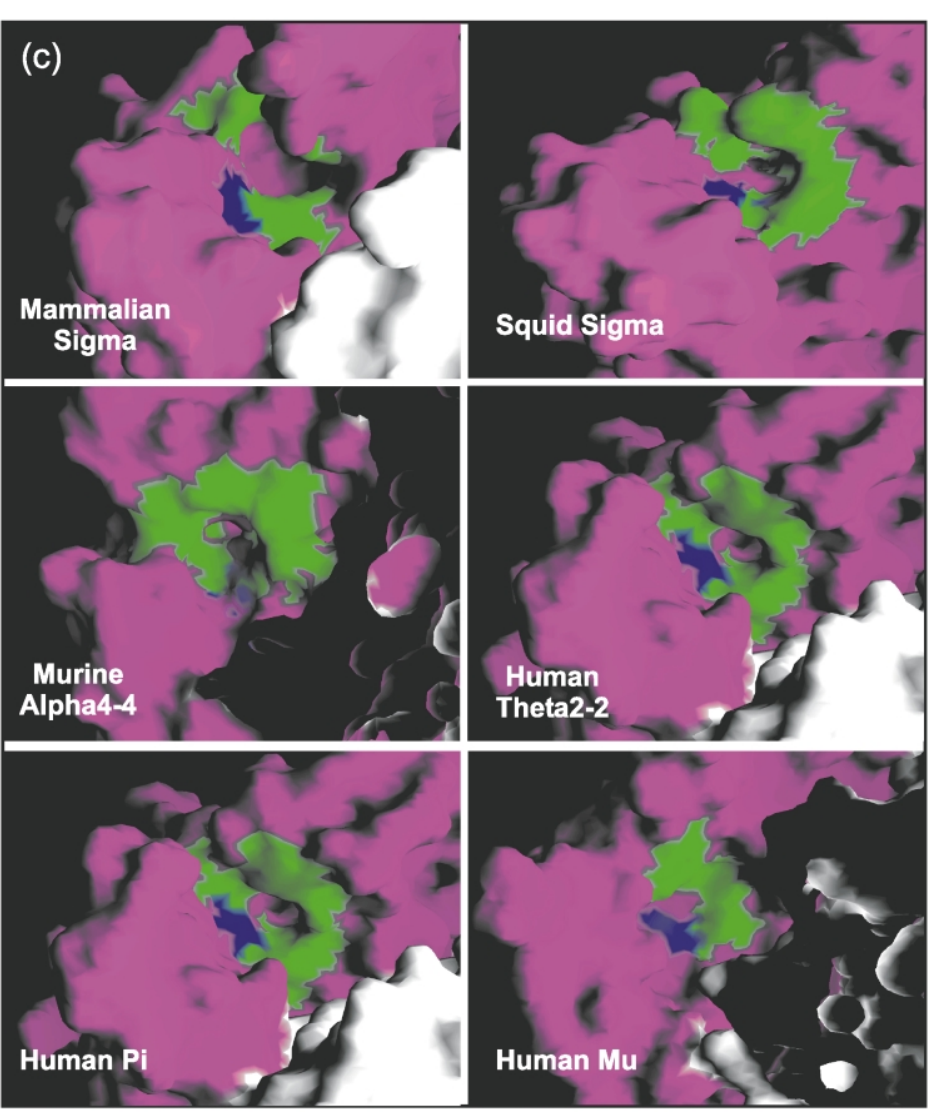

Figure 4. Topology of the GST-2 H-site in comparison to other GSTs. (a) Stereo view showing the relative position of $\alpha 6$ helix in GST-2 compared to selected GSTs. The comparison was based on a 3D-structure superposition using only the N-terminal domains, excluding the structurally variant helix $\alpha 2$. Helix $\alpha 1$ is shown as a reference point, together with Y208. Thick black line, GST-2; thin black line, rat Sigma (PDB: 1PD2); medium-broken black line, squid Sigma (1GSQ); highly broken black line, mouse Alpha4-4 (1B48); thick gray line, human Mu (1GTU); thin gray line, human Pi (1PGT); thick broken gray line, human Theta (1LJR). (b) The putative H-site residues of GST2 shown in green in ball and stick (right panel) and in yellow in surface representation (left panel). In both panels the catalytic residue Y54 is colored cyan. The bound GSH in the G-site is also shown (left panel). The surface of monomer A (excluding the H-site and Y54) is colored in magenta, while monomer B is shown in white. (c) Active site surface of GSTs from selected classes, indicating residues largely forming the H-site pocket (in green) and the catalytic residue (in blue). Protein Data Bank coordinates were as described in the legend to Figure 1. The pockets are defined by residues R13, F106, F98 and F202 for the squid Sigma, ${ }^{38}$ R14, W104, Y152 and L199 for the mammalian Sigma, ${ }^{6}$ L12, H107, F208 and Y115 for the human Mu, ${ }^{84}$ R107, V10 and W115 for the human Theta, ${ }^{29}$ R13, V104, Y108 and V10 for the human Pi and R15, M104, V108, A107, V116, G14, Y212 and F111 for the murine Alpha4- $4^{37}$ GSTs. 

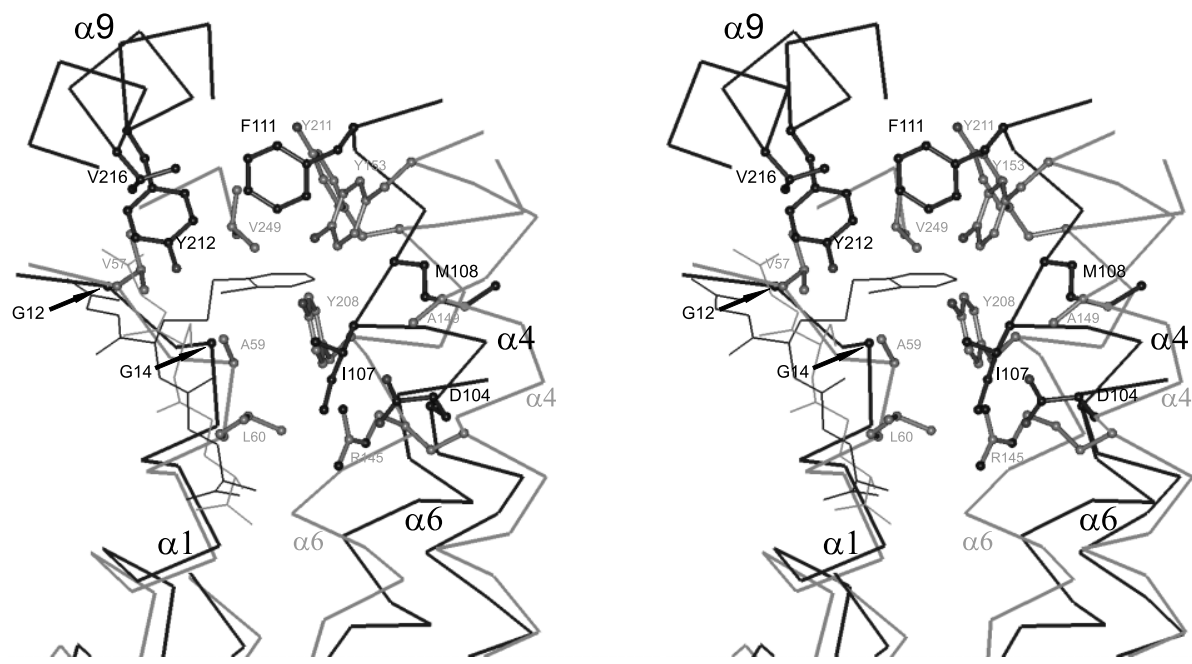

Figure 5. Comparison of the H-sites of GST-2 and human Alpha4-4 GST. The $\mathrm{C}^{\alpha}$-trace of both molecules and the putative H-site residues of GST-2 and electrophilic-binding residues (in ball and stick) of hAlpha4-4 are shown in gray and black, respectively. Various secondary structure elements were omitted for clarity. GSH bound to GST-2 is shown as a bonds model in gray. The Alpha4-4 GST structure used (PDB: 1GUL) was solved in complex with the inhibitor $S$-(2-iodobenzyl)-glutathione, ${ }^{42}$ which is shown as a black bonds model. The two structures were superimposed using only their N-terminal domains (excluding helix $\alpha 2$ ).

a largely hydrophobic cavity which binds the hydrophobic moiety of the electrophilic co-substrate (Figure 4(c)). A superposition of the C-terminal domain of GST-2 onto the equivalent domains of GSTs from other classes reveals how this is accomplished: helix $\alpha 6$ of GST-2 is displaced towards the G-site (Figure 4(a)), resulting in the swing of the side-chain of Y208 so that it occupies the space that would otherwise be a cavity. Indicative of this movement is the $C^{\alpha}-C^{\alpha}$ distance of Y208 to the equivalent residues in the squid and vertebrate Sigma-GSTs and the mouse Alpha4-4 GST, which is $3 \AA, 3 \AA$ and $7.5 \AA$, respectively. The deviation of the $\alpha 6$ helix is provoked by a bulge at approximately the middle of the helix at G204, a residue that is not found in that position in other GSTs (Figure 1(a) and not shown sequence alignments). The beginning of helix $\alpha 6$ is stabilized by an N-capping box and a weak hydrophobic staple interaction, motifs that are present in all soluble GSTs known. ${ }^{43}$

\section{Comparison to Sigma, insect Delta and Alpha4-4 H-sites}

The Sigma ${ }^{7,44}$ and insect Delta ${ }^{15,45}$ isoenzymes are orders of magnitude more active than GST-2 towards the model substrate CDNB. Instead, GST2 is catalytically closer to the vertebrate Alpha4-4 subclass. Since this contrast in activity is most likely originating from diversity in the H-sites of the enzymes, we performed a structural comparison of the H-site of GST-2 and the H-sites of the squid $^{38}$ and vertebrate ${ }^{6}$ Sigma and rat and human Alpha4-4 structures. ${ }^{37,42}$ We also compared the active site of GST-2 with the ones from the three insect Delta structures solved to date, from L. cuprina $^{32}$ and from A.dirus (AdGST1-3 and
AdGST1-4). ${ }^{33}$ The polar nature of the H-site in GST-2 shows similarities with the polar base of the $\mathrm{H}$-site in the Delta structures. In particular, residues Y105 and Y113 in the Lucilia and AdGST1-3 (Y111 and Y119 in AdGST1-4) structures are oriented similarly to Y208 and Y153 of GST-2. However, at least in the case of the Anopheles enzymes, there is an adjacent small hydrophobic pocket formed by Y105 (Y111), Y113 (Y119) and hydrophobic residues from the C-terminal $\alpha 8$ helix (this helix is missing in the Lucilia structure), which might explain their high activity against CDNB. Both the Delta and GST-2 H-sites are solvent-exposed and open towards the $C$ terminus. The comparison with the Sigma homologs reveals that, in contrast to GST-2, the Sigma structures feature prominent hydrophobic H-site pockets, a deep and wide one in the vertebrate Sigma (formed by residues R14, Y152, L199 and W104) and a narrower one in the squid Sigma (defined by residues F98, R13, F106, and F202). Additionally, the Sigma sites are significantly less polar compared to GST-2. Nonetheless, both pockets are open. Finally, there are significant differences in the Alpha4-4 H-sites as well: first, the presence of helix $\alpha 9$ (Figure 1(a)) in these enzymes occludes access to the active site; second, the topography is not flat, with a notable hydrophobic cavity formed by residues G14, I107, F111, V216 and Y212 (Figure 5). Y212 of human Alpha4-4 has been implicated in contacting the aldehydic oxygen of 4 -HNE based on a protein-inhibitor complex structure. ${ }^{42}$ The functional equivalent of Y212 in GST-2 is residue Y208. Indeed, when we superimposed the G-sites of the inhibitor complex structure (1GUL) and GST-2 the iodine of the inhibitor comes at a distance of $3.4 \AA$ from the hydroxyl of Y208 and $7.15 \AA$ from the $\mathrm{N}^{n}$ nitrogen of R145 (Figure 5). In 


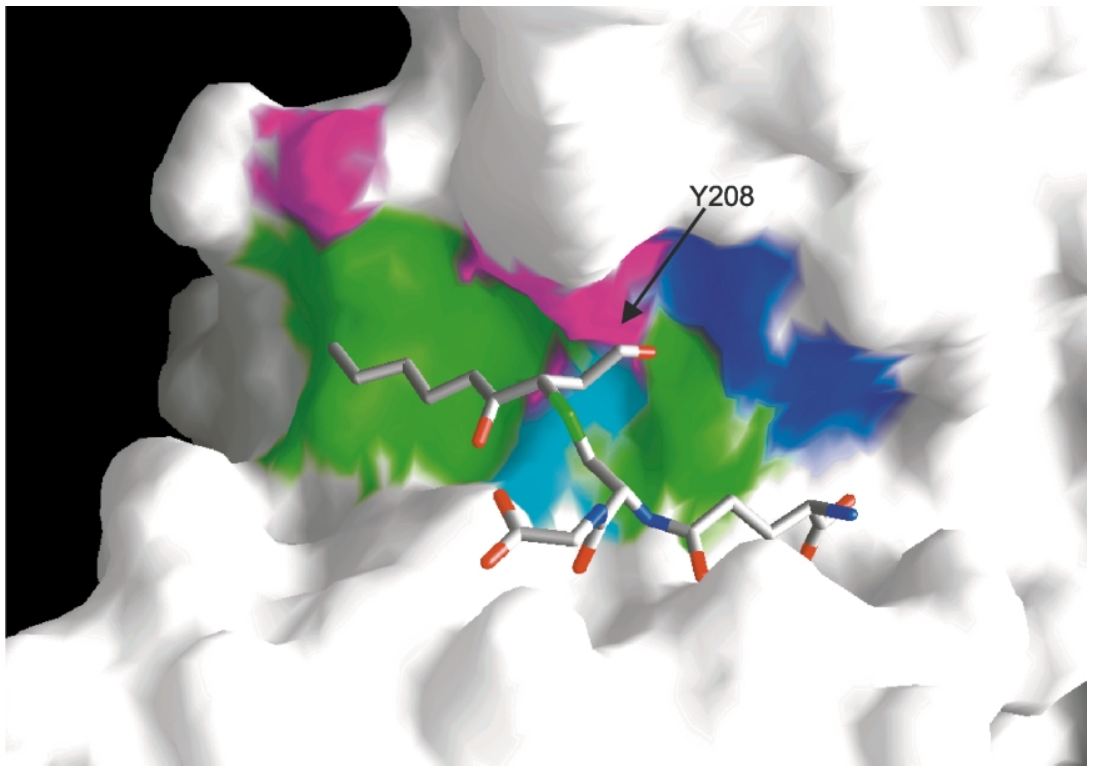

Figure 6. Model of 4-HNE binding to GST-2. A sticks representation of the GS-HNE conjugate in a putative-binding position in the GST-2 active site is shown (for details see the text). Surface patch coloring: green, hydrophobic sidechains; magenta, tyrosine hydroxyls; blue, guanidino group; cyan, hydroxyl of the catalytic tyrosine Y54. The surface corresponding to the side-chain carbon atoms of Y153 and Y211 is not colored for clarity. a similar superimposition with a mouse Alpha4-4HNE complex (1B48), the aldehydic oxygen of 4HNE was within hydrogen bonding distance to both $\mathrm{O}^{\eta}$ of Y208 $(2.8 \mathrm{~A})$ and $\mathrm{N}^{\eta}$ of R154 (3.1 A). However, in both cases, the inhibitor and the aliphatic part of 4-HNE clash with both Y208 and Y153. This is in accordance with GST-2 being a poor enzyme against electrophilic compounds harboring aromatic rings and also suggests that GST-2 would probably bind 4-HNE in a different orientation than the Alpha4-4 enzymes.

\section{A model for 4-HNE binding}

A structure-based model for the binding of the 4-HNE conjugate to GST-2 was constructed based on: (i) the proposed role of Y208 and R145 in catalysis, (ii) the orientation of GSH in our structure, (iii) the fact that the GSH sulfur attacks carbon-3 of 4-HNE in the reaction and (iv) manual fitting to obtain optimum interactions between the co-substrate's hydrophobic chain and the hydrophobic residues of the H-site (Figure 6). A model of 4-HNE bound to human Alpha4-4 ${ }^{42}$ indicated that R15, the equivalent of R145 in GST-2, mediates the protonation of carbon- 2 of 4 -HNE to complete the breakage of the 2-alken double bond. In our model the $\mathrm{N}^{\eta}$ nitrogen of $\mathrm{R} 145$ is $5.8 \AA$ from carbon-2, suggesting that this arginine is not performing the same function in GST-2. R145 is also too far to interact with the substrate's hydroxyl group. Our model is consistent with polarization of the aldehydic oxygen by the hydroxyl oxygen of Y208 (O-O distance, $2.85 \AA)$. The carbonyl oxygen is also close $(3.6 \mathrm{~A})$ to the $\mathrm{N}^{n}$ of R145, indicating that the guanidino group of this arginine may also contribute to the polarization. It is noteworthy that the carbonyl oxygen in the model is $2.3 \AA$ from the W2 water molecule (Figure 3 ) and that both chiralities about the hydroxyl-bearing carbon- 4 can be accommodated. In some substrate orientations it is possible for the 4-hydroxyl oxygen to come close to the hydroxyl of Y153. It cannot therefore be excluded that Y153 if slightly shifted may also contact 4-HNE. Finally, the hydrophobic tail packs against residues A59, V57 and V249 in accord with their putative role in the H-site.

\section{Discussion}

Insect Sigma GSTs are a poorly characterized group among the GST superfamily, distinct in their phylogenetic position. Interestingly, GST-2 from Drosophila and the house fly (M. domestica) differentiate even more by having a proline/ alanine-rich N-terminal extension. The physiological role of this extension is not totally clear, but it might be used in the Drosophila IFM to attach the protein to the thin filament. The attachment of GST-2 to troponin led Bullard and colleagues ${ }^{20}$ to suggest that GST-2 could have a role in "stretch activation", an intriguing phenomenon, whereby a small amount of stretching activates contraction of the flight muscle. ${ }^{46}$ The hypothesis was strengthened by the fact that the Musca ortholog had a similar tissue distribution. ${ }^{47}$ However, the fact that some $(\sim 10-20 \%)$ portion of GST-2 in both Drosophila and Musca is in non-muscular tissues and that the N-terminal extension is missing from other insect Sigma GSTs ${ }^{48,49}$ challenges the hypothesis. The N-terminal extension is disordered in the GST-2 crystal structure, suggesting that it does not associate strongly with the rest of the molecule, in contrast to the $\mathrm{N}$-extension of human Omega GST, which folds back to form a continuous surface with the $\mathrm{C}$ terminus. ${ }^{50}$

The catalytic specificity of GST-2 was established by Singh et al. $^{22}$ and confirmed, in part, here. The enzyme shows a clear preference for elongated unsaturated compounds with carbonyl oxygen atoms, i.e. slim compounds of amphipolar nature. The structure of the H-site provides an explanation 
for this; the site is shallow and due to the special geometry of the $\alpha 6$ helix there is no hydrophobic pocket that could optimally accommodate a small aromatic ring. Furthermore, there is directionality in the surface distribution of polar groups, with Y153, Y208 and R145 on one side (close to the catalytic Y54) and a hydrophobic band at the other. As shown in Figure 6, this topology is consistent with the geometry and polarity of 4-HNE and in fact fixes the carbonyl oxygen and C-3 carbon in a favorable positions for catalysis. An arginine residue in the vicinity of the catalytic tyrosine has been implicated in assisting catalysis in GSTs from many classes, including both 1-1 and 4-4 Alpha subclasses ${ }^{37,42,51}$ and the Sigma class. ${ }^{44}$ In the insect Delta structures ${ }^{32,33}$ no evidence of such a functional arginine exists. In our structure, R145 has a distance to the sulfydryl group of GSH compatible with a role in the catalytic mechanism. It is important to note that R145 resides on helix $\alpha 4$ while in other cases the equivalent arginine is on the $\beta 1-\alpha 1$ loop. This indicates the flexibility of GSTs to correctly position functional groups irrespective of whether they come from the $\mathrm{C}$ or N-terminal domain. A similar usurpation has been observed in a comparison of Alpha structures, where helix $\alpha 9$ in Alpha4-4 swaps function with the $\beta 1-\alpha 1$ loop of Alpha1-1. ${ }^{42}$ Analogously, the functional equivalence of Y212 in Alpha4-4 and Y208 in GST2 , as suggested by our structure, demonstrates a functional swapping between $\mathrm{H}$-site residues within the C-terminal domain. Mutations of residue Y212 in Alpha4-4 dramatically affected the specificity towards 4-HNE and $\mathrm{EA}^{42}$ and towards trans-2-nonenal, ${ }^{41}$ suggesting that Y208 in GST-2 could behave the same. In that respect, it would be of interest to target this residue in mutational analyses. The structure suggests that Y153 could also be involved in catalysis. A major role for Y153 is questioned by the fact that its hydroxyl was, in most modelling attempts, far from the HNE moiety (Figure 6) and from a previous study showing that mutation of the equivalent residue (Y113) in L. cuprina did not affect GSH activation. ${ }^{52}$ In the squid Sigma, however, substitution of the cognate phenylalanine residue (F106) with tyrosine dramatically improved the efficiency of the enzyme towards enone and epoxide substrates. ${ }^{38}$ Thus, experimental evidence is required to resolve the role of Y153 in GST-2.

The enzymatic data presented by Singh et al. ${ }^{22}$ demonstrated that GST-2 is at least 20-times less efficient in processing 4-HNE compared to the mammalian Alpha4-4 isoenzymes. In addition, the enzyme is comparable in its glutathione peroxidase activity and in some cases in its activity towards trans-2-nonenal to some of the insect Delta GSTs. ${ }^{50,53}$ However, vertebrate Sigma GSTs are significantly less effective against trans-2-nonenal although some are only two to threefold less active towards 4-HNE than GST-2. ${ }^{54}$ Although these variations cannot be fully explained from the GST2 structure, at least in the case of alk-2-enals a plausible explanation could be the absence of the deep hydrophobic groove seen in the Alpha4-4 enzymes (made largely by helix $\alpha 9$ ), that optimally fits the long hydrophobic chains of these compounds. Paradoxically, we found that the aryl halide $\mathrm{NBD}-\mathrm{Cl}$ is a slightly better substrate than trans-2-nonenal. This compound has a bulkier planar moiety than CDNB and is more polar, which possibly makes it more suitable for the polar GST-2 H-site. In relative terms, however, GST-2 is not a competent enzyme against NBD-Cl either, as the observed activity is orders of magnitudes lower compared to the avian Sigma GST ${ }^{54}$ and other GSTs.

The sequence, catalytic properties and tissue distribution of GST-2 in Drosophila suggest a distinctly different function for this enzyme in comparison to the Delta class GSTs of this organism, which are implicated in general detoxification and confer resistance towards insecticides. This is supported by the differences in the active site structures (see Results) and from the fact that most residues that line the H-site residues in GST-2 are conserved within the insect Sigma class but vary compared to the Delta class. As elegantly discussed by Zimniak and colleagues, ${ }^{22}$ this role could be in protecting the muscle or other sensitive tissues like the neuronal tissues from the deleterious products of oxidative stress, of which 4-HNE is among the most dangerous. ${ }^{25}$ Such a role complies with recent studies linking Drosophila GSTs to antioxidant defence ${ }^{55,56}$ and longevity. ${ }^{57-60}$ While in most of these studies a detailed identification of the GSTs involved was not performed, at least in one case a genomic screen identified GST-2 as being essential for the extension of life span of Drosophila..$^{8}$

Insect IFMs contain one of the highest numbers of mitochondria per cell unit volume found in nature, which, because of the high contraction frequencies, are required to work intensively and thus release large amounts of oxygen reactive species into the intracellular space. ${ }^{61,62}$ In addition, recent studies show that insect flight mitochondrial proteins are highly prone to oxidative damage. ${ }^{63,64}$ Thus, given the close proximity of mitochondria to the myofibril, a detoxification role for GST-2 could be physiologically relevant. This is supported by the astonishing abundance of GST-2, which accounts for about two-thirds of the total GST mass in Drosophila (and $\sim 70 \%$ of the total 4 -HNE conjugating activity). ${ }^{22}$ It is evident, however, that further experimental work is necessary to delineate the function of GST-2 in the muscle for which the structure presented here provides a basis.

\section{Materials and Methods}

\section{Recombinant protein expression, purification and crystallization}

Recombinant GST-2 was expressed, purified and crystallized as described. ${ }^{65}$ When not used for crystallization, 
Table 2. Summary of X-ray data, refinement and phasing statistics

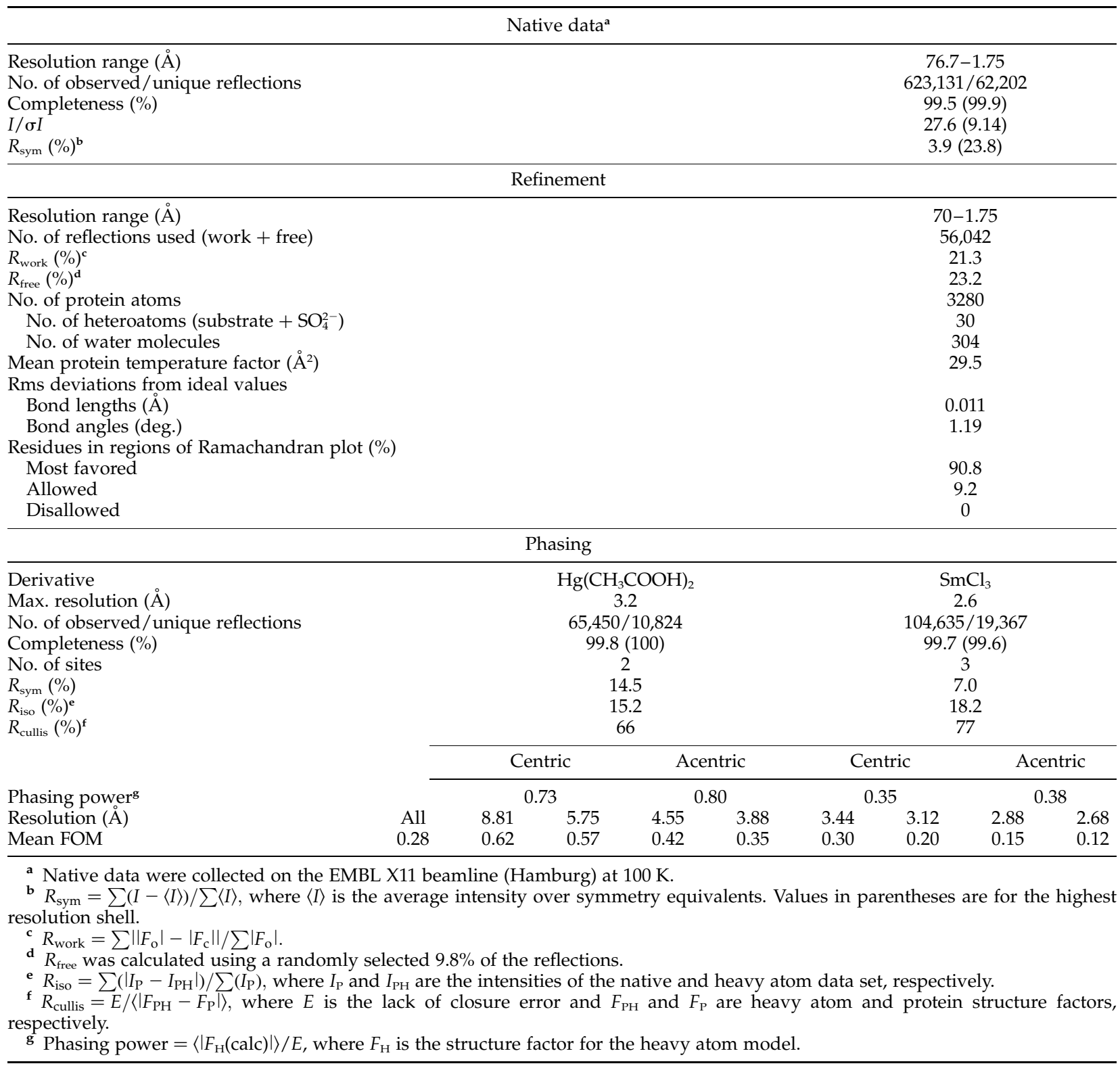

aliquots of the protein in $20 \mathrm{mM}$ sodium phosphate $(\mathrm{pH}$ 7.0), $2 \mathrm{mM} \beta$-mercaptoethanol, $15 \mathrm{mM}$ GSH (storage buffer) were stored at $-80^{\circ} \mathrm{C}$ for future use. To confirm that intact GST-2 was crystallized, a single big crystal was extensively washed with reservoir solution, dissolved in water and the solution was subjected to MALDI-MS analysis.

\section{Enzymatic assays}

All GST enzymatic activity assays were followed spectrophotometrically at $22^{\circ} \mathrm{C}$. Non-enzymatic contribution was subtracted using control reactions in which GST-2 was substituted with buffer. Activities towards 1-chloro2,4-dinitrobenzol (CDNB), 1,2-dichloro-4-nitrobenzene (DCNB) and ethacrynic acid (EA) were performed as described. ${ }^{66}$ Conjugation of GSH to 7-chloro-4-nitrobenzo-2-oxa-1,3-diazole (NBD-Cl) was measured as described by Ricci et al. ${ }^{34}$ To assess the effect of $\mathrm{TnH}$, the
NBD-Cl assay was repeated in the presence of $\mathrm{TnH}$ in up to 2:1 molar ratio to GST-2. The activity towards trans-2-nonenal was determined using a standard assay, ${ }^{67}$ except using the modifications in substrate concentrations presented by Hubatsch et al. ${ }^{26}$

\section{Sequence alignment and phylogenetic tree construction}

For the structure-based alignment, the amino acid sequences of a subgroup of GSTs with known 3D-structures were aligned using the program Clustal $\mathrm{X}^{68}$ The alignment was manually adjusted to optimize correspondence of equivalent structural elements and minimize gaps. A bigger group of sequences, which included representatives of the major GST classes including insect GSTs, was separately aligned. Distances between each pair of the aligned sequences and an unrooted phylogenetic 
tree were calculated within Clustal X. The bootstrap values at the tree notes were calculated using 1000 bootstrap repetitions.

\section{Heavy atom derivative preparation}

Heavy atom derivative crystals were obtained by soaking native GST-2 crystals for 16 hours in $200 \mu l$ of mother liquor containing the derivative. The end concentration of both successful derivatives $\mathrm{Hg}\left(\mathrm{CH}_{3} \mathrm{COOH}\right)_{2}$ and $\mathrm{SmCl}_{3}$ in the soaking solution was $2 \mathrm{mM}$.

\section{Data collection and processing}

Derivative crystals were transferred to a cryoprotection solution composed of soaking solution saturated in sucrose and were flash frozen to $100 \mathrm{~K}$ before data collection. X-ray data were collected on Beamline ID14-EH4 of the European Synchrotron Radiation Facility (ESRF, Grenoble, France), using a MAR CCD detector. The wavelength $\lambda$ used was $0.9392 \AA$ and the oscillation step per diffraction frame $1^{\circ}$. All data were indexed, merged and scaled with the DENZO and SCALEPACK programs. ${ }^{69}$

\section{Structure determination, model building and refinement}

The structure was determined using multiple isomorphous replacement with anomalous scattering (MIRAS) as implemented in the program SOLVE. ${ }^{70}$ Despite the poor phasing statistics (Table 2), density modification within CNS_solve v $1.0^{71}$ improved the mean FOM from 0.28 to 0.73 and gave an interpretable experimental electron density map. Most of the polypeptide chain was automatically built using ARP/wARP ${ }^{72}$ followed by repeated cycles of manual building using O. ${ }^{73}$ Refinement was performed using initially CNS and finally REFMAC. ${ }^{74}$ Residues $1-46$ and the side-chain of D154 from both monomers were disordered and weak density was observed for the loop residues 153-156 of monomer A and 153-159 of monomer B. Water molecules were built using $\mathrm{ARP}^{75}$ followed by manual inspection based on $2 F_{\mathrm{o}}-F_{\mathrm{c}}$ and $F_{\mathrm{o}}-F_{\mathrm{c}} \sigma_{\mathrm{A}}$ weighted maps. Finally, two sulfate ions and one GSH molecule were fitted in a $F_{\mathrm{o}}-$ $F_{\mathrm{c}}$ composite omit map using phases from the protein atoms alone. The density for GSH in the G-site of monomer A was weak but interpretable and the ligand was fitted at the position that gave the highest real-space correlation and agreed with conserved GSH-binding interactions observed in other GST structures. The GSH density in monomer B was extremely weak and discontinuous, indicating positional disorder and/or low occupancy of the ligand, despite the presence of GSH excess $(15 \mathrm{mM})$ in the crystallization conditions. ${ }^{65}$ Therefore, GSH was not modeled in monomer B. During the final refinement steps disconnected density appeared in the vicinity of GSH and at the inter-subunit interface which, however, remained uninterpretable. Investigation of crystal packing does not exclude the possibility that the inter-subunit density could account for parts of the disordered N-terminal extension. Validity of the model was checked using $R_{\text {free }}$ and omit maps. Despite the presence of non-crystallographic symmetry, NCS constraints were not used throughout. The Ramachandran plot indicates no residues in disallowed regions and shows that residues A59, M94 and Q109 have strained conformations. Final refinement statistics are summarized in Table 2.
Structural quality of the model was verified using PRO$\mathrm{CHECK}^{76}$ and WHATIF. $^{77}$ Figures were produced using ALSCRIPT, $^{78}$ MOLSCRIPT, $^{79}$ BOBSCRIPT $^{80}$ and GRASP. ${ }^{81}$

\section{Atomic coordinates}

The atomic coordinates have been deposited in the Protein Data Bank under PDB code 1M0U.

\section{Acknowledgements}

We thank the staff at the ESRF synchrotron in Grenoble, in particular R.B.G. Ravelli, and the beamline staff at the EMBL Hamburg outstation for support in data collection. We are grateful to S.H.W. Scheres for assistance.

\section{References}

1. Hayes, J. D. \& Pulford, D. J. (1995). The glutathione S-transferase supergene family: regulation of GST and the contribution of the isoenzymes to cancer chemoprotection and drug resistance. Crit. Rev. Biochem. Mol. Biol. 30, 445-600.

2. Board, P. G., Baker, R. T., Chelvanayagam, G. \& Jermiin, L. S. (1997). Zeta, a novel class of glutathione transferases in a range of species from plants to humans. Biochem. J. 328, 929-935.

3. Kanaoka, Y., Fujimori, K., Kikuno, R., Sakaguchi, Y., Urade, Y. \& Hayaishi, O. (2000). Structure and chromosomal localization of human and mouse genes for hematopoietic prostaglandin D synthase. Conservation of the ancestral genomic structure of sigmaclass glutathione S-transferase. Eur. J. Biochem. 267, 3315-3322.

4. Ketterman, A. J., Prommeenate, P., Boonchauy, C., Chanama, U., Leetachewa, S., Promtet, N. \& Prapanthadara, L. (2001). Single amino acid changes outside the active site significantly affect activity of glutathione S-transferases. Insect Biochem. Mol. Biol. $31,65-74$.

5. Board, P. G., Coggan, M., Chelvanayagam, G., Easteal, S., Jermiin, L. S., Schulte, G. K. et al. (2000). Identification, characterization, and crystal structure of the Omega class glutathione transferases. J. Biol. Chem. 275, 24798-24806.

6. Kanaoka, Y., Ago, H., Inagaki, E., Nanayama, T., Miyano, M., Kikuno, R. et al. (1997). Cloning and crystal structure of hematopoietic prostaglandin D synthase. Cell, 90, 1085-1095.

7. Jowsey, I. R., Thomson, A. M., Flanagan, J. U. Murdock, P. R., Moore, G. B., Meyer, D. J. et al. (2001). Mammalian class Sigma glutathione S-transferases: catalytic properties and tissue-specific expression of human and rat GSH-dependent prostaglandin D2 synthases. Biochem. J. 359, 507-516.

8. Tomarev, S. I., Zinovieva, R. D., Guo, K. \& Piatigorsky, J. (1993). Squid glutathione S-transferase. Relationships with other glutathione S-transferases and S-crystallins of cephalopods. J. Biol. Chem. 268, 4534-4542.

9. Liebau, E., Walter, R. D. \& Henkle-Duhrsen, K. (1994). Isolation, sequence and expression of an 
Onchocerca volvulus glutathione S-transferase cDNA. Mol. Biochem. Parasitol. 63, 305-309.

10. Meyer, D. J., Muimo, R., Thomas, M., Coates, D. \& Isaac, R. E. (1996). Purification and characterization of prostaglandin-H E-isomerase, a sigma-class glutathione S-transferase, from Ascaridia galli. Biochem. J. 313, 223-227.

11. Tomarev, S. I. \& Zinovieva, R. D. (1988). Squid major lens polypeptides are homologous to glutathione S-transferases subunits. Nature, 336, 86-88.

12. Syvanen, M., Zhou, Z. H. \& Wang, J. Y. (1994). Glutathione transferase gene family from the housefly Musca domestica. Mol. Gen. Genet. 245, 25-31.

13. Ranson, H., Rossiter, L., Ortelli, F., Jensen, B., Wang, X., Roth, C. W. et al. (2001). Identification of a novel class of insect glutathione S-transferases involved in resistance to DDT in the malaria vector Anopheles gambiae. Biochem. J. 359, 295-304.

14. Beall, C., Fyrberg, C., Song, S. \& Fyrberg, E. (1992). Isolation of a Drosophila gene encoding glutathione S-transferase. Biochem. Genet. 30, 515-527.

15. Fournier, D., Bride, J. M., Poirie, M., Berge, J. B. \& Plapp, F. W., Jr (1992). Insect glutathione S-transferases. Biochemical characteristics of the major forms from houseflies susceptible and resistant to insecticides. J. Biol. Chem. 267, 1840-1845.

16. Reiss, R. A. \& James, A. A. (1993). A glutathione S-transferase gene of the vector mosquito, Anopheles gambiae. Insect Mol. Biol. 2, 25-32.

17. Chelvanayagam, G., Parker, M. W. \& Board, P. G. (2001). Fly fishing for GSTs: a unique nomenclature for mammalian and insect glutathione transferases. Chem. Biol. Interact. 133, 256-260.

18. Hemingway, J. (2000). The molecular basis of two contrasting metabolic mechanisms of insecticide resistance. Insect Biochem. Mol. Biol. 30, 1009-1015.

19. Prapanthadara, L., Promtet, N., Koottathep, S., Somboon, P. \& Ketterman, A. J. (2000). Isoenzymes of glutathione S-transferase from the mosquito Anopheles dirus species B: the purification, partial characterization and interaction with various insecticides. Insect Biochem. Mol. Biol. 30, 395-403.

20. Clayton, J. D., Cripps, R. M., Sparrow, J. C. \& Bullard, B. (1998). Interaction of troponin-H and glutathione S-transferase-2 in the indirect flight muscles of Drosophila melanogaster. J. Muscle Res. Cell Motil. 19, 117-127.

21. Bullard, B., Leonard, K., Larkins, A., Butcher, G., Karlik, C. \& Fyrberg, E. (1988). Troponin of asynchronous flight muscle. J. Mol. Biol. 204, 621-637.

22. Singh, S. P., Coronella, J. A., Benes, H., Cochrane, B. J. \& Zimniak, P. (2001). Catalytic function of Drosophila melanogaster glutathione S-transferase DmGSTS1-1 (GST-2) in conjugation of lipid peroxidation end products. Eur. J. Biochem. 268, 2912-2923.

23. Zarkovic, N., Zarkovic, K., Schaur, R. J., Stolc, S., Schlag, G., Redl, H. et al. (1999). 4-Hydroxynonenal as a second messenger of free radicals and growth modifying factor. Life Sci. 65, 1901-1904.

24. Ji, C., Amarnath, V., Pietenpol, J. A. \& Marnett, L. J. (2001). 4-Hydroxynonenal induces apoptosis via caspase-3 activation and cytochrome c release. Chem. Res. Toxicol. 14, 1090-1096.

25. Esterbauer, H., Schaur, R. J. \& Zollner, H. (1991). Chemistry and biochemistry of 4-hydroxynonenal, malonaldehyde and related aldehydes. Free Radic. Biol. Med. 11, 81-128.

26. Hubatsch, I., Ridderstrom, M. \& Mannervik, B. (1998). Human glutathione transferase A4-4: an alpha class enzyme with high catalytic efficiency in the conjugation of $\mathrm{n}$ and other genotoxic products of lipid peroxidation. Biochem. J. 330, 175-179.

27. Zimniak, P., Eckles, M. A., Saxena, M. \& Awasthi, Y. C. (1992). A subgroup of class alpha glutathione S-transferases. Cloning of cDNA for mouse lung glutathione S-transferase GST 5.7. FEBS Letters, 313, 173-176.

28. Sheehan, D., Meade, G., Foley, V. M. \& Dowd, C. A. (2001). Structure, function and evolution of glutathione transferases: implications for classification of non-mammalian members of an ancient enzyme superfamily. Biochem. J. 360, 1-16.

29. Rossjohn, J., Polekhina, G., Feil, S. C., Allocati, N., Masulli, M., De Illio, C. \& Parker, M. W. (1998). A mixed disulfide bond in bacterial glutathione transferase: functional and evolutionary implications. Structure, 6, 721-734.

30. Reinemer, P., Prade, L., Hof, P., Neuefeind, T., Huber, R., Zettl, R. et al. (1996). Three-dimensional structure of glutathione S-transferase from Arabidopsis thaliana at $2.2 \AA$ resolution: structural characterization of herbicide-conjugating plant glutathione S-transferases and a novel active site architecture. J. Mol. Biol. 255, 289-309.

31. Neuefeind, T., Huber, R., Reinemer, P., Knablein, J., Prade, L., Mann, K. \& Bieseler, B. (1997). Cloning, sequencing, crystallization and X-ray structure of glutathione S-transferase-III from Zea mays: a leading enzyme in detoxification of maize herbicides. J. Mol. Biol. 274, 577-587.

32. Wilce, M. C., Board, P. G., Feil, S. C. \& Parker, M. W. (1995). Crystal structure of a theta-class glutathione transferase. EMBO J. 14, 2133-2143.

33. Oakley, A. J., Harnnoi, T., Udomsinprasert, R., Jirajaroenrat, K., Ketterman, A. J. \& Wilce, M. C. (2001). The crystal structures of glutathione S-transferases isozymes 1-3 and 1-4 from Anopheles dirus species B. Protein Sci. 10, 2176-2185.

34. Ricci, G., Caccuri, A. M., Lo Bello, M., Pastore, A., Piemonte, F. \& Federici, G. (1994). Colorimetric and fluorometric assays of glutathione transferase based on 7-chloro-4-nitrobenzo-2-oxa-1,3-diazole. Anal. Biochem. 218, 463-465.

35. Armstrong, R. N. (1997). Structure, catalytic mechanism, and evolution of the glutathione transferases. Chem. Res. Toxicol. 10, 2-18.

36. Thom, R., Dixon, D. P., Edwards, R., Cole, D. J. \& Lapthorn, A. J. (2001). The structure of a zeta class glutathione S-transferase from Arabidopsis thaliana: characterisation of a GST with novel active-site architecture and a putative role in tyrosine catabolism. J. Mol. Biol. 308, 949-962.

37. Xiao, B., Singh, S. P., Nanduri, B., Awasthi, Y. C., Zimniak, P. \& Ji, X. (1999). Crystal structure of a murine glutathione S-transferase in complex with a glutathione conjugate of 4-hydroxynon-2-enal in one subunit and glutathione in the other: evidence of signaling across the dimer interface. Biochemistry, 38, 11887-11894.

38. Ji, X., von Rosenvinge, E. C., Johnson, W. W., Tomarev, S. I., Piatigorsky, J., Armstrong, R. N. \& Gilliland, G. L. (1995). Three-dimensional structure, catalytic properties, and evolution of a sigma class glutathione transferase from squid, a progenitor of the lens S-crystallins of cephalopods. Biochemistry, 34, 5317-5328.

39. Dirr, H., Reinemer, P. \& Huber, R. (1994). X-ray crystal structures of cytosolic glutathione S-transferases. 
Implications for protein architecture, substrate recognition and catalytic function. Eur. J. Biochem. 220, 645-661.

40. Ji, X., Blaszczyk, J., Xiao, B., O’Donnell, R., Hu, X., Herzog, C. et al. (1999). Structure and function of residue 104 and water molecules in the xenobiotic substrate-binding site in human glutathione S-transferase P1-1. Biochemistry, 38, 10231-10238.

41. Hubatsch, I. \& Mannervik, B. (2001). A highly acidic tyrosine 9 and a normally titrating tyrosine 212 contribute to the catalytic mechanism of human glutathione transferase A4-4. Biochem. Biophys. Res. Commun. 280, 878-882.

42. Bruns, C. M., Hubatsch, I., Ridderstrom, M., Mannervik, B. \& Tainer, J. A. (1999). Human glutathione transferase A4- 4 crystal structures and mutagenesis reveal the basis of high catalytic efficiency with toxic lipid peroxidation products. J. Mol. Biol. 288, 427-439.

43. Aceto, A., Dragani, B., Melino, S., Allocati, N., Masulli, M., Di Ilio, C. \& Petruzzelli, R. (1997). Identification of an $\mathrm{N}$-capping box that affects the alpha 6-helix propensity in glutathione S-transferase superfamily proteins: a role for an invariant aspartic residue. Biochem. J. 322, 229-234.

44. Pinzar, E., Miyano, M., Kanaoka, Y., Urade, Y. \& Hayaishi, O. (2000). Structural basis of hematopoietic prostaglandin D synthase activity elucidated by sitedirected mutagenesis. J. Biol. Chem. 275, 31239-31244.

45. Jirajaroenrat, K., Pongjaroenkit, S., Krittanai, C., Prapanthadara, L. \& Ketterman, A. J. (2001). Heterologous expression and characterization of alternatively spliced glutathione S-transferases from a single Anopheles gene. Insect Biochem. Mol. Biol. 31, 867-875.

46. Pringle, J. W. (1978). The Croonian Lecture, 1977. Stretch activation of muscle: function and mechanism. Proc. R. Soc. Lond. B. Biol. Sci. 201, 107-130.

47. Franciosa, H. \& Berge, J. B. (1995). Glutathione S-transferases in housefly (Musca domestica): location of GST-1 and GST-2 families. Insect Biochem. Mol. Biol. 25, 311-317.

48. Arruda, L. K., Vailes, L. D., Platts-Mills, T. A., Hayden, M. L. \& Chapman, M. D. (1997). Induction of IgE antibody responses by glutathione S-transferase from the German cockroach (Blattella germanica). J. Biol. Chem. 272, 20907-20912.

49. Snyder, M. J., Walding, J. K. \& Feyereisen, R. (1995). Glutathione S-transferases from larval Manduca sexta midgut: sequence of two cDNAs and enzyme induction. Insect Biochem. Mol. Biol. 25, 455-465.

50. Board, P., Russell, R. J., Marano, R. J. \& Oakeshott, J. G. (1994). Purification, molecular cloning and heterologous expression of a glutathione S-transferase from the Australian sheep blowfly (Lucilia cuprina). Biochem. J. 299, 425-430.

51. Krengel, U., Schroter, K. H., Hoier, H., Arkema, A., Kalk, K. H., Zimniak, P. \& Dijkstra, B. W. (1998). Crystal structure of a murine alpha-class glutathione S-transferase involved in cellular defense against oxidative stress. FEBS Letters, 422, 285-290.

52. Caccuri, A. M., Antonini, G., Nicotra, M., Battistoni, A., Bello, M. L., Board, P. G. et al. (1997). Catalytic mechanism and role of hydroxyl residues in the active site of theta class glutathione S-transferases. Investigation of Ser-9 and Tyr-113 in a glutathione S-transferase from the Australian sheep blowfly, Lucilia cuprina. J. Biol. Chem. 272, 29681-29686.
53. Lee, H. C. \& Tu, C. P. (1995). Drosophila glutathione S-transferase D27: functional analysis of two consecutive tyrosines near the $\mathrm{N}$-terminus. Biochem. Biophys. Res. Commun. 209, 327-334.

54. Thomson, A. M., Meyer, D. J. \& Hayes, J. D. (1998). Sequence, catalytic properties and expression of chicken glutathione-dependent prostaglandin D2 synthase, a novel class Sigma glutathione S-transferase. Biochem. J. 333, 317-325.

55. Missirlis, F., Phillips, J. P. \& Jackle, H. (2001). Cooperative action of antioxidant defense systems in Drosophila. Curr. Biol. 11, 1272-1277.

56. Fernandez-Funez, P., Nino-Rosales, M. L., de Gouyon, B., She, W. C., Luchak, J. M., Martinez, P. et al. (2000). Identification of genes that modify ataxin-1-induced neurodegeneration. Nature, 408, 101-106.

57. Le Bourg, E. (2001). Oxidative stress, aging and longevity in Drosophila melanogaster. FEBS Letters, 498, 183-186.

58. Seong, K. H., Ogashiwa, T., Matsuo, T., Fuyama, Y. \& Aigaki, T. (2001). Application of the gene search system to screen for longevity genes in Drosophila. Biogerontology, 2, 209-217.

59. Toba, G. \& Aigaki, T. (2000). Disruption of the microsomal glutathione S-transferase-like gene reduces life span of Drosophila melanogaster. Gene, 253, 179-187.

60. Zou, S., Meadows, S., Sharp, L., Jan, L. Y. \& Jan, Y. N. (2000). Genome-wide study of aging and oxidative stress response in Drosophila melanogaster. Proc. Natl Acad. Sci. USA, 97, 13726-13731.

61. Han, D., Williams, E. \& Cadenas, E. (2001). Mitochondrial respiratory chain-dependent generation of superoxide anion and its release into the intermembrane space. Biochem. J. 353, 411-416.

62. Droge, W. (2002). Free radicals in the physiological control of cell function. Physiol. Rev. 82, 47-95.

63. Yan, L. J. \& Sohal, R. S. (1998). Mitochondrial adenine nucleotide translocase is modified oxidatively during aging. Proc. Natl Acad. Sci. USA, 95, 12896-12901.

64. Das, N., Levine, R. L., Orr, W. C. \& Sohal, R. S. (2001). Selectivity of protein oxidative damage during aging in Drosophila melanogaster. Biochem. J. 360, 209-216.

65. Agianian, B., Clayton, J. D., Leonard, K., Tucker, P., Bullard, B. \& Gros, P. (2001). Crystallization and preliminary X-ray analysis of Drosophila glutathione S-transferase-2. Acta Crystallog. sect. D, 57, 725-727.

66. Habig, W. H., Pabst, M. J. \& Jakoby, W. B. (1974). Glutathione S-transferases. The first enzymatic step in mercapturic acid formation. J. Biol. Chem. 249, 7130-7139.

67. Brophy, P. M., Southan, C. \& Barrett, J. (1989). Glutathione transferases in the tapeworm Moniezia expansa. Biochem. J. 262, 939-946.

68. Jeanmougin, F., Thompson, J. D., Gouy, M., Higgins, D. G. \& Gibson, T. J. (1998). Multiple sequence alignment with Clustal X. Trends Biochem. Sci. 23, 403-405.

69. Otwinowski, A. \& Minor, W. (1997). Processing of X-ray diffraction data collected in oscillation mode. Methods Enzymol. 276, 307-326.

70. Terwilliger, T. C. \& Berendzen, J. (1999). Automated MAD and MIR structure solution. Acta Crystallog. sect. $D, 55,849-861$.

71. Brunger, A. T., Adams, P. D., Clore, G. M., DeLano, W. L., Gros, P., Grosse-Kunstleve, R. W. et al. (1998). Crystallography \& NMR system: a new software suite for macromolecular structure determination. Acta Crystallog. sect. D, 54, 905-921. 
72. Perrakis, A., Morris, R. \& Lamzin, V. S. (1999). Automated protein model building combined with iterative structure refinement. Nature Struct. Biol. 6, $458-463$.

73. Jones, T. A., Zou, J. Y., Cowan, S. W. \& Kjeldgaard, M. (1991). Improved methods for binding protein models in electron density maps and the location of errors in these models. Acta Crystallog. sect. A, 47, 110-119.

74. Murshudov, G. N., Vagin, A. A., Lebedev, A., Wilson, K. S. \& Dodson, E. J. (1999). Efficient anisotropic refinement of macromolecular structures using FFT. Acta Crystallog. sect. D, 55, 247-255.

75. Collaborative Computing Project Number 4 (1994). The CCP4 suite: programs for protein crystallography. Acta Crystallog. sect. D, 50, 760-763.

76. Laskowski, R. A., MacArthur, M. W., Moss, D. S. \& Thornton, J. M. (1993). PROCHECK: a program to check the stereochemical quality of protein structures. J. Appl. Crystallog. 26, 283-291.

77. Vriend, G. (1990). WHAT IF: a molecular modeling and drug design program. J. Mol. Graph. 8, 52-56.

78. Barton, G. J. (1993). ALSCRIPT: a tool to format multiple sequence alignments. Protein Eng. 6, 37-40.
79. Kraulis, P. J. (1991). MOLSCRIPT: a program to produce both detailed and schematic plots of protein structures. J. Appl. Crystallog. 24, 946-950.

80. Esnouf, R. M. (1999). Further additions to MolScript version 1.4, including reading and contouring of electron-density maps. Acta Crystallog. sect. D, 55, 938-940.

81. Honig, B. \& Nicholls, A. (1995). Classical electrostatics in biology and chemistry. Science, 268, 1144-1149.

82. Livingstone, C. D. \& Barton, G. J. (1993). Protein sequence alignments: a strategy for the hierarchical analysis of residue conservation. Comput. Appl. Biosci. 9, 745-756.

83. Wallace, A. C., Laskowski, R. A. \& Thornton, J. M. (1995). LIGPLOT: a program to generate schematic diagrams of protein-ligand interactions. Protein Eng. 8, 127-134.

84. Patskovsky, Y. V., Patskovska, L. N. \& Listowsky, I. (1999). Functions of His107 in the catalytic mechanism of human glutathione S-transferase hGSTM1a-1a. Biochemistry, 38, 1193-1202.

Edited by R. Huber

(Received 2 September 2002; received in revised form 6 November 2002; accepted 8 November 2002) 